\title{
Substructure and Signs of Planet Formation in the Disk of HD 169142
}

\author{
M. Osorio ${ }^{1}$, G. Anglada ${ }^{1}$, C. Carrasco-González ${ }^{2,3}$, J. M. Torrelles ${ }^{4}$, \\ P. D’Alessio ${ }^{3}$, L. F. Rodríguez ${ }^{3}$, N. Calvet ${ }^{5}$, J. F. Gómez ${ }^{1}$, \\ J. M. Mayen-Gijon ${ }^{1}$ and W. R. F. Dent ${ }^{6}$ \\ ${ }^{1}$ Instituto de Astrofísica de Andalucía (CSIC), Camino Bajo de Huétor 50, \\ E-18008 Granada, Spain email: osorio@iaa.es \\ ${ }^{2}$ Max-Planck-Institut für Radioastronomie, Auf dem Hügel 69, 53121 Bonn, Germany \\ ${ }^{3}$ Centro de Radioastronomía y Astrofísica, UNAM, \\ 58089 Morelia, Michoacán, Mexico \\ ${ }^{4}$ Instituto de Ciencias del Espacio (CSIC) - UB/IEEC, \\ Martí i Franquès 1, E-08028 Barcelona, Spain \\ ${ }^{5}$ Department of Astronomy, University of Michigan, \\ 825 Dennison Building, 500 Church St, Ann Arbor, MI 48109, USA \\ ${ }^{6}$ ALMA SCO, Alonso de Córdova 3107, Vitacura, Santiago, Chile
}

\begin{abstract}
We carried out $7 \mathrm{~mm}$ VLA observations at very high angular resolution that reveal substructure and evidence of planet formation in the disk of HD 169142. Our observations, along with near-infrared polarimetric imaging, show that this disk has a ring of enhanced, asymmetric emission at a radius of $\sim 25 \mathrm{AU}$ from the central star. This ring, whose inner region appears devoid of emission, is surrounded by an annular gap in surface density in the $\sim 30-70$ AU range of radii. Several mechanisms have been invoked in the literature to explain this kind of gaps and cavities. Among them, one of the most interesting is the possibility that one or more planets in formation are creating these cavities. Since our $7 \mathrm{~mm}$ observations show a compact source lying in the 30-70 AU gap, we speculate that this compact source could be tracing dust emission associated with a possible protoplanet. We model the broad-band spectral energy distribution of the disk and we infer its physical structure. From this modeling we infer the presence of a small $(r \sim 0.7 \mathrm{AU}$ ) disk inside the central cavity, suggesting that the HD 169142 disk is in the pre-transitional disk phase.
\end{abstract}

Keywords. stars: formation, planetary systems: formation, protoplanetary disks

\section{Introduction}

Transitional disks are protoplanetary disks that have developed central cavities of several tens AU in size almost devoid of dust but full of gas since the central star is still accreting (Calvet et al. 2005). A subclass of these disks, the so-called pre-transitional disks, with a remaining inner disk inside the central cavity, was introduced to account for significant near-infrared excesses observed in a number of objects (Espaillat et al. 2008). Several mechanisms have been invoked to explain these central cavities such as photoevaporation (Alexander et al. 2006), grain-growth (Dullemond \& Dominik 2004), or planetary formation (Augereau \& Papaloizou 2004). If planets were actually opening these cavities, then transitional and pre-transitional disks would be the best sites to search for planets in the verge of their formation.

The presence of most of these cavities has been indirectly inferred through the modeling of the Spitzer spectra. But in the last years an increasing number have been angularly resolved by interferometers like the Submillimeter Array (SMA; see Andrews et al. 2012) 
and the Atacama Large Millimeter/submillimeter Array (ALMA; see Casassus et al. 2013).

An interesting case of study is the protoplanetary disk around the Herbig Ae/Be star HD 169142. HD 169142 is an intermediate-mass star $\left(\sim 2 M_{\odot}\right)$ located at a distance of 145 pc. Scattered light images as well as molecular observations reveal a face-on disk extending up to $250 \mathrm{AU}$. Dent et al. (2006) detected relatively strong, angularly unresolved $7 \mathrm{~mm}$ emission through Very Large Array (VLA) observations. Since centimeter emission (Dent et al. 2006; Osorio et al. 2013) is not detected towards this object, it is expected that the observed $7 \mathrm{~mm}$ emission traces the dust emission from the disk, without free-free emission contamination from a possible radio jet. The lack of detectable free-free emission also suggests that this object is in a relatively advanced evolutionary stage where the accretion and outflow processes are almost halted. On the other hand, the observed SED does not exhibit a silicate feature at 10 microns, a result that is suggestive of significant grain growth, a process required for planetary formation.

The presence of an inner cavity in the HD 169142 disk was inferred from the modeling of the observed spectral energy distribution (SED). From these models, estimates of $\sim 40$ AU (Grady et al. 2007) and 20 AU (Meeus et al. 2010; Honda et al. 2012) were obtained for the radius of this central cavity. Confirmation of a central cavity has been obtained recently through NACO/VLT polarized light images by Quanz et al. (2013) (gray-scale image in Fig. 1). In addittion to a central cavity with a radius of $\sim 20 \mathrm{AU}$, these observations reveal a bright ring located at a radius of $\sim 25 \mathrm{AU}$ and an annular gap extending from $\sim 30$ to $70 \mathrm{AU}$.

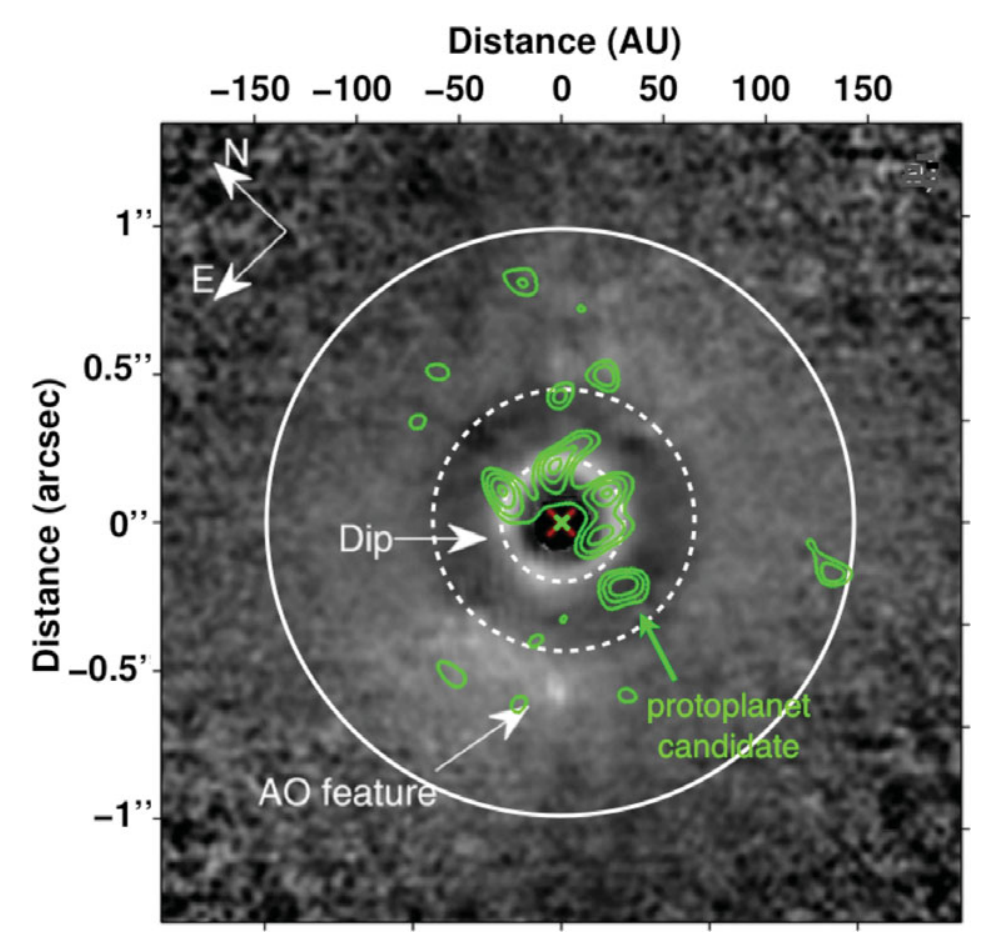

Figure 1. VLA map at $7 \mathrm{~mm}$ (contours; Osorio et al. 2013) overlaid on the VLT/NACO polarized light image in the $\mathrm{H}$ band (gray-scale; from Quanz et al. 2013). The size of the VLA beam is $\sim 0.2^{\prime \prime}$. Saturated pixels in the central region of the H-band image have been masked out. The cross marks the 2MASS position of the HD 169142 star, after correction for proper motions to the epoch of the observations (2012). 


\section{VLA Observational Results}

VLA observations at $7 \mathrm{~mm}$ and $6 \mathrm{~cm}$ were carried out in the $\mathrm{CnB}$ and $\mathrm{A}$ configurations along 2012 (Osorio et al. 2013). No emission was detected at $6 \mathrm{~cm}$, indicating that there is no significant free-free emission associated with this object. In Figure 1 (contours) we show a preliminary map of the combined $\mathrm{CnB}+\mathrm{A}$ data. The $7 \mathrm{~mm}$ map, with an angular resolution of $\sim 0.2^{\prime \prime}$, shows a (partial) ring-like structure that coincides pretty well with the ring observed in the polarized infrared image obtained by Quanz et al. (2013). The ring is asymmetric, with the weakest part coinciding with a dip observed in the polarized light image. Outside the ring, at a radius of $43 \mathrm{AU}$, there is an unresolved $7 \mathrm{~mm}$ source (0.15 mJy) that falls within the annular gap observed by Quanz et al. (2013). We speculate that this $7 \mathrm{~mm}$ source could be tracing circumplanetary dust associated with a young protoplanet. Assuming a temperature of $100-300 \mathrm{~K}$, a $7 \mathrm{~mm}$ opacity of 0.9 $5 \times 10^{-3} \mathrm{~cm}^{-2} \mathrm{~g}^{-1}$, and a gas-to-dust mass ratio of 100 , a mass of $0.2-2 \mathrm{M}_{J}$ is obtained. If the $7 \mathrm{~mm}$ source traces the protoplanet that has created the 30-70 AU annular gap, its orbital period would be $186 \mathrm{yr}$, and we expect significant proper motions in a few years.

\section{Modeling of the Disk}

We modeled the Spectral Energy Distribution (SED) of the circumstellar disk of HD 169142 using the accretion disk models developed by D'Alessio et al. (1999, 2001, 2006). Following the observations, we have truncated the disk at an inner radius of $20 \mathrm{AU}$, and we considered that the disk is extending out to $250 \mathrm{AU}$. In this model the annular gap located between 30 and $70 \mathrm{AU}$ is not considered because it is expected to have a small effect on the SED, as it appears to be partially filled (see Quanz et al. 2013) and the temperatures expected at these distances are relatively low. We included the emission of an inner wall at a radius of $20 \mathrm{AU}$ with a temperature of $200 \mathrm{~K}$, calculated from the equilibrium equation, assuming a stellar luminosity of $17 L_{\odot}$. However, this model does not reproduce the near-infrared range of the observed SED (dotted line in Fig. 2). To fit this wavelength range, we included a small central disk of 0.7 AU radius, with a wall heated at a temperature of $1200 \mathrm{~K}$ (the dust sublimation temperature) located at $0.4 \mathrm{AU}$, its inner radius. By adding these components to the previous model we are able to explain the broad-band SED and the $7 \mathrm{~mm}$ image. This model (thick solid line in Fig. 2) is in agreement with the constrains set by the VLA observations. It predicts weak $7 \mathrm{~mm}$ emission (undetectable with the sensitivity achieved with the VLA) inside a radius of $20 \mathrm{AU}$, because the small central disk and its wall radiate most of the emission in the 2-10 $\mu \mathrm{m}$ wavelength range (see Fig. 2). Note that the central region was masked out in the H-band observations of Quanz et al. (2013); so, these infrared observations cannot be used to constrain the size of the small inner disk.

\section{Summary and Proposed Scenario for HD 169142}

We obtained a high angular resolution $\left(0.2^{\prime \prime}\right)$ VLA image at $7 \mathrm{~mm}$ of the inner structure of the HD 169142 protoplanetary disk. The morphology of the $7 \mathrm{~mm}$ emission is well correlated with the VLT infrared scattered light image obtained by Quanz et al. (2013), showing a ring of radius $\sim 25$ AU surrounded by an annular gap from $\sim 30$ to $70 \mathrm{AU}$.

The SED fitting and the VLA/VLT data indicate that the HD 169142 disk is a pretransitional disk, with a small inner disk, from 0.4 to $0.7 \mathrm{AU}$, and an outer disk from 20 to 250 AU. There is, therefore, a gap from 0.7-20 AU, and a second, probably less developed, gap extending from 30-70 AU. 


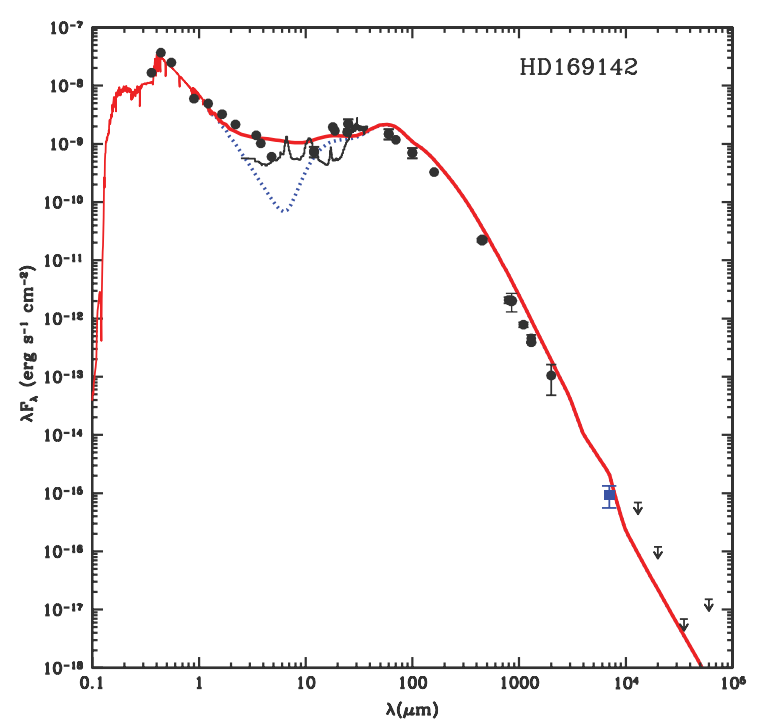

Figure 2. Observed (points and thin line) and model (dotted and thick solid lines) SEDs of the HD 169142 protoplanetary disk. The thick solid line corresponds to a model including the small $(\sim 0.7 \mathrm{AU})$ central disk and its wall, while the dotted line corresponds to a model without this central disk (see text).

Inside the 30-70 AU annular gap we detect a $7 \mathrm{~mm}$ unresolved source, that we tentatively interpret as tracing the dust associated with a protoplanet candidate. We estimate a total mass of $0.2-2 \mathrm{M}_{J}$ for this proposed circumplanetary structure.

We interpret these results as evidence of planetary formation in the disk of HD 169142. This hypothesis can be further tested with new VLA, VLT, and ALMA observations.

\section{Acknowledgements}

GA, CC-G, JFG, JMM-G, MO, and JMT acknowledge support from MINECO (Spain) AYA2008-06189-C03 and AYA2011-30228-C03 grants, co-funded with FEDER funds.

\section{References}

Alexander, R. D., Clarke, C. J., \& Pringle, J. E. 2006, MNRAS, 369, 216

Andrews, S. M., Rosenfeld, K. A., Wilner, D. J., \& Bremer, M. 2011, ApJ, 742, L5

Augereau, J. C. \& Papaloizou, J. C. B. 2004, A\& A, 414, 1153

Calvet, N., D'Alessio, P., Watson, D. M., et al. 2005, ApJ (Letters), 630, L185

Casassus, S., van der Plas, G., M, S. P., et al. 2013, Nature, 423, 191

D’Alessio, P., Calvet, N., Hartmann, L., Lizano, S., \& Cantó, J. 1999, ApJ, 527, 893

D'Alessio, P., Calvet, N., \& Hartmann, L. 2001, ApJ, 553, 321

D’Alessio, P., Calvet, N., Hartmann, L., Franco-Hernández, R., \& Servín, H. 2006, ApJ, 638, 314

Dullemond, C. P. \& Dominik, C. 2004, A\& A, 421, 1075

Espaillat, C., Calvet, N., Luhman, K. L., Muzerolle, J., \& D'Alessio, P. 2008, ApJ (Letters), 682, L125

Grady, C. A., Schneider, G., Hamaguchi, K., et al. 2007, ApJ, 665, 1391

Honda, M., Maaskant, K., Okamoto, Y. K., et al. 2012, ApJ, 752, 143

Meeus, G., Pinte, C., Woitke, P., et al. 2010, A\& A, 518, L124

Osorio, M., et al. 2013, in preparation

Quanz, S. P., Avenhaus, H., Buenzli, E., et al. 2013, ApJ (Letters), 766, L2 